\title{
Protease nexin-1 prevents growth of human B cell lymphoma via inhibition of sonic hedgehog signaling
}

\author{
Xiangke Xin ${ }^{1}$, Yunchuan Ding ${ }^{2}$, Ying Yang $\mathbb{1}^{1}$, Xing Fu', Jianfeng Zhou' ${ }^{1}$ Chad M. McKee ${ }^{3}$, Ruth J. Muschel ${ }^{3}$, \\ Robert P. Gale, ${ }^{4}$ Jane F. Apperley ${ }^{4}$ and Danmei $X u^{1,4}$
}

Diffuse large B cell lymphoma (DLBCL) is the most common non-Hodgkin lymphoma (NHL) in adults ${ }^{1}$. Onethird to half of people with DLBCL is not cured despite substantial therapy advance ${ }^{2}$. The variability of outcome is associated with prognostic scoring system such as the International Prognostic Index (IPI), which focus on biological features of the host and of the neoplastic cells ${ }^{1}$. However, considerable recent data suggest lymphoma microenvironment contributes to prognosis by affecting lymphoma cell survival and angiogenesis ${ }^{3-5}$. For example, gene chip from DLBCL tissues revealed a stromal signature that associated with favorable prognosis ${ }^{5}$. This signature is predominantly composed of genes encoding extracellular matrix (ECM) components and modifiers such as laminin, fibronectin, and collagen ${ }^{5}$. Those with a stromal 2 pattern encoding angiogenesis-related genes are unfavorable ${ }^{5}$.

Matrix metalloproteinase-9 (MMP-9) regulates tumor microenvironment by degrading ECM and collagen typeIV, disrupting the physical barrier to facilitate cancer cell invasion $^{6}$. MMP9 is over-expressed in high-grade NHL, associated with an unfavorable prognosis in DLBCL ${ }^{6,7}$. SB3CT, a potent gelatinase inhibitor against MMP-9/ MMP-2, inhibits angiogenesis, prevents formation of lymphatic vessels, and reduces the spread of lymphoma cells in a mouse $\mathrm{T}$ cell lymphoma model ${ }^{8}$. However, little is known of how MMP-9 regulates the lymphoma microenvironment.

\footnotetext{
Correspondence: Danmei Xu (danmei.xu@nhs.net)

'Department of Haematology, Tongji Hospital, Tongji Medical College,

Huazhong University of Science and Technology, Hubei, China

${ }^{2}$ Department of Endocrinology, Tongji Hospital, Tongji Medical College,

Huazhong University of Science and Technology, Hubei, China
}

Full list of author information is available at the end of the article
In a prior study through a proteomic approach, we showed that protease nexin-1 (PN-1) is susceptible to MMP-9-mediated degradation in the prostate cancer microenvironment ${ }^{9}$. $\mathrm{PN}-1$, a $43 \mathrm{kDa}$ serine protease inhibitor, prevents several key micro-environmental proteases including urokinase plasminogen activator (uPA, encoded by $P L A U$ ), tissue plasminogen activator (tPA), thrombin, and plasmin ${ }^{10,11}$. High levels of MMP-9 and uPA are associated with disease progression and an unfavorable prognosis in several human solid cancers ${ }^{11}$. Although deregulation of PN-1 occurs in several solid cancers, the role if any, of PN-1 in hematological cancers is unknown. The coincidence of MMP9 and PLAU gene found in the DLBCL stromal 1 signature ${ }^{5}$ suggests a possible link between expression of these genes and the lymphoma microenvironment.

First, low levels of PN-1 were found in lymphoid tissues (lymph nodes and spleen) infiltrated with DLBCL cells compared with controls (Fig. 1a). High transcript levels of $M M P 9$ were found in the DLBLC-infiltrated tissues, consistent with prior reports and gene chip data ${ }^{5}$. In contrast, PN1 transcript levels in DLBCL-infiltrated tissues were significantly lower compared with reactive lymph nodes (Fig. 1b). Interestingly, in contrast to the low transcript levels of PN1 in the DLBCL-infiltrated bone marrow, bone marrow from subjects with DLBCL without bone marrow involvement had high levels of PN1 transcripts (Fig. 1c). Thus, PN-1 levels appear reduced in B cell lymphoma.

To determine how PN-1 affects the biology of lymphoma cells, we examined endogenous PN1 transcript levels in a number of human $B$ cell lymphoma cell lines (Figure S1A). In contrast to low PN1 expression, $M M P 9$ transcript levels were highest in Raji cells (derived from 


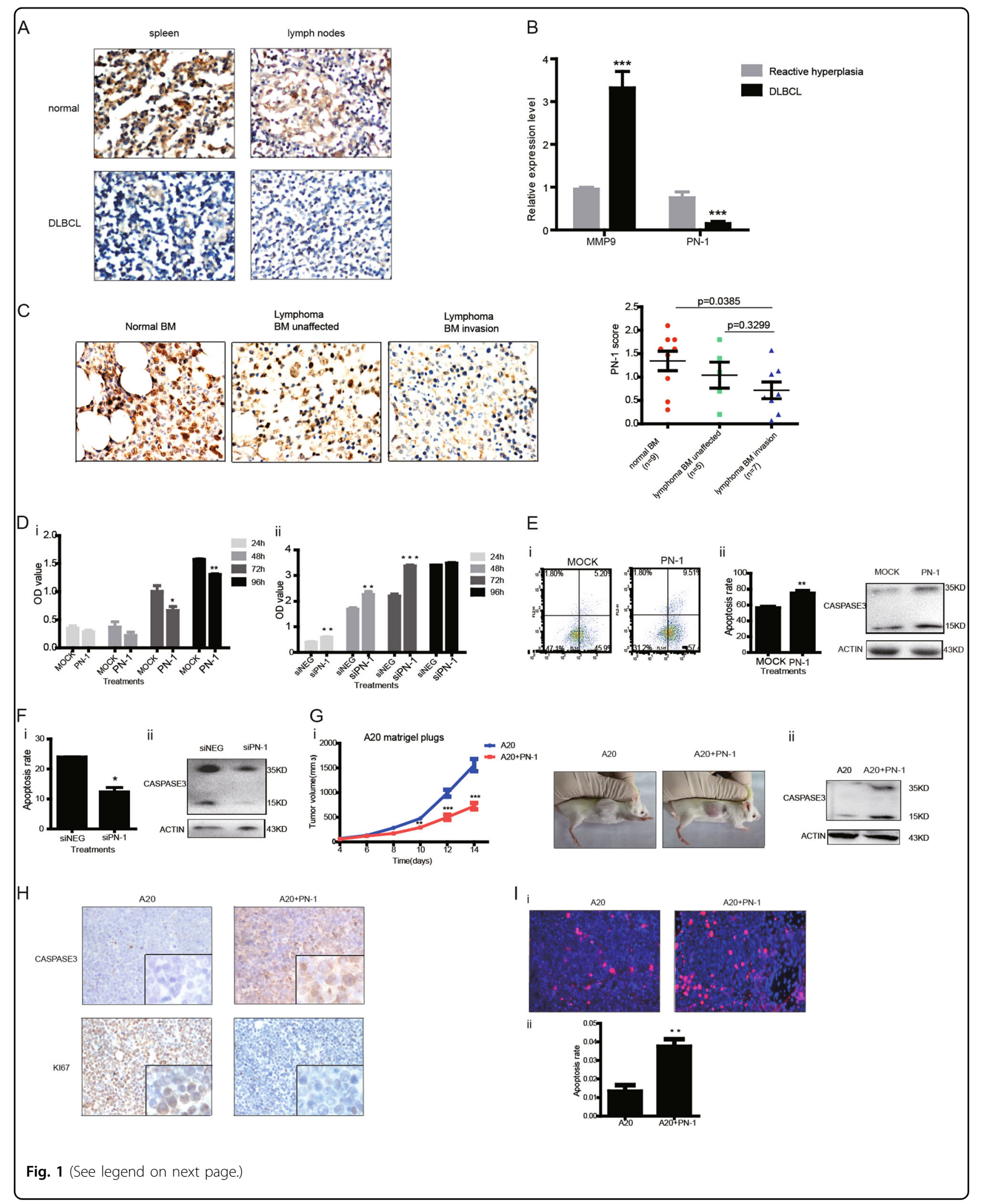




\begin{abstract}
Fig. 1 The expression pattern of PN-1 in DLBCL and its functional impact on lymphoma cell proliferation and apoptosis. a Immune histochemical staining (brown) of PN-1 in spleen and lymph nodes from normals or infiltrated with DLBCL cells. Blue stain represents haemotoxylin nuclear staining. $\mathbf{b}$ Quantitative real-time PCR for the expression of MMP9 and PN1 in lymph nodes hyperplasia (LN) and DLBCL. $N=6$, ***P<0.001. Student's $t$ test was performed for statistical significance. c DAB staining of PN-1 (brown, panel i) and relative expression score (ii) in controls with a normal bone marrow $(N=9)$, persons with lymphoma with an involved $(N=7)$ or uninvolved bone marrow $(N=6)$. One-way ANOVA performed for statistical significance. $\mathbf{d}$ Proliferation of Raji cells $\left(2 \times 10^{6}\right)$ was analyzed by using CCK-8 assays at 24, 48, 72, and $96 \mathrm{~h}$ after transfection with $2 \mu \mathrm{g}$ of pcDNA3-PN1 or control plasmid (i) or followed by treatment of 40 pmol siRNA PN1 (siPN1) or control scrambled siRNA (siNEG) $(N=3$; ttest * $P<0.05$; ${ }^{*} P<0.01$; ${ }^{* *} P<0.001$ ); e Apoptosis analysis was determined by Annexin-PI assay (i) and immune-blotting of cleavage of caspase-3 (15-kDa active band) (ii) in Raji cells transfected with $2 \mu \mathrm{g}$ of pcDNA3-PN1 or control plasmid ( $N=4$; ttest ${ }^{* *} P<0.01$ ); $\mathbf{f}$ Raji cells transfected with siRNA PN1 (siPN1) or control siRNA (siNEG) were assayed for apoptosis $\left(N=3 ; t\right.$ test $\left.{ }^{*} P<0.05\right) ; \mathbf{g}$ Subcutaneous tumor volumes from Balb/c mice implanted with A20 cells $\left(1 \times 10^{6}\right)$ mixed with Matrigel with or without PN-1 recombinant protein $(10 \mu \mathrm{M})$. Subcutaneous tumor growth curve (i) $\left(N=5 ;\right.$; test, ${ }^{* *} P<0.01$; ${ }^{* * *} P<$ 0.001 ) and tumor volumes (ii); immune-blotting detecting caspase-3 cleavage (iii); $\mathbf{h}$ A20 xenografts with or without pre-treatment with PN-1 (10 $\mu \mathrm{M})$ recombinant protein, DAB-stained (brown) for caspase-3 and Ki67. Blue stain represents hematoxylin nuclear staining. (i) in situ apoptotic assay using TUNEL. Positive cells (red stain) were counted from three microscopic fields and plotted $\left(N=4, t\right.$ test, $\left.{ }^{*} P<0.01\right)$. Nuclei are blue stained
\end{abstract}

Burkitt lymphoma) (Figure S1B). In contrast, Wsunhl and Jeko cells (derived from follicular lymphoma and mantle cell lymphoma respectively) with lower $M M P 9$ transcript levels than Raji cells had high levels of PN1 transcripts (Figure S1B), in keeping with a known regulation of PN-1 being a substrate target of MMP-99,12,13. Adding exogenous PN-1 prevented proliferation of Raji cells and A20 cells (a mouse B cell lymphoma cell line) while knockdown of PN1 expression by siRNA increased proliferation of these cells (Figs. 1di-ii and S2A). Adding exogenous PN1 to Raji and A20 cells increased apoptosis determined by flow cytometry and by the caspase- 3 assay (Figs. 1e and S2B). In contrast, apoptosis decreased in cells where PN1 expression was reduced by siRNA (Fig. 1f).

Next, we developed a syngeneic B cell lymphoma mouse model by subcutaneous injection of A20 cells into Balb/c mice. Adding recombinant $\mathrm{PN}-1$ protein $(10 \mu \mathrm{M})$ to the Matrigel plug significantly delayed lymphoma growth (Fig. 1gi). Tumor volumes of mice treated with exogenous $\mathrm{PN}-1$ protein after 12 days were $500 \mathrm{~mm}^{3}$ compared with $989 \mathrm{~mm}^{3}$ in controls $(p<0.001)$. Apoptosis rates in the tumor grafts were examined in situ using a TUNEL assay and/or immune blotting or immune-histochemistry for caspase-3 cleavage (Fig. 1gi). Adding PN-1-induced apoptosis while inhibiting proliferation as evidenced by markedly increased caspase-3 staining and decreased Ki67 staining (Fig. 1h).

The $S H H$-signaling pathway is activated in DLBCL and contributes to lymphoma cell survival and proliferation ${ }^{14}$. Over-expression of PN1 resulted in a reduced transcription and protein expression of molecules in the $\mathrm{SHH}$ signaling pathway including SHH, GLI1, and PTCH1 in human Raji and mouse A20 cells (Figs. 2a and S2C). Conversely, PN1 downregulation by siRNA was associated with increased expression of SHH and GLI1 (Fig. 2b), indicating PN1 expression inhibits $S H H$ signaling in lymphoma cells. Here, we compared inhibitory effects of PN-1 on lymphoma cells with those of the $S H H$ signaling inhibitor cyclopamine, which irreversibly binds SMO and inactivates SHH signaling. Raji cells treated with exogenous PN1 or $10 \mu \mathrm{M}$ cyclopamine can both prevent SHH-signaling transcription (Fig. 2c). There was more inhibition of SHH signaling when cells were treated with PN1 plasmid transfection and cyclopamine compared with cyclopamine alone (Fig. 2d). Increasing concentrations of cyclopamine produced a dose-dependent decrease of mRNA levels of GLI1 and SHH (Figure S3A). Adding PN-1 recombinant protein had a similar effect as cyclopamine in reducing GLII and $S H H$ expression in A20 cells (Figure S3B-C). At 48 and $72 \mathrm{~h}$, cyclopamine was a stronger inhibitor of proliferation, whereas effects on apoptosis were similar (Fig. 2e). We also determined $S H H$ and GLI1 transcript levels in A20 cells treated with recombinant PN-1. Levels of $S H H$ and GLI1 transcripts were reduced by 96.8 and $86.8 \%$ after treatment with $100 \mathrm{ng} / \mathrm{ml}$ recombinant PN-1 (Fig. 2f). Apoptosis of A20 cells induced by recombinant PN-1 was similar to that induced by cyclopamine (Figure S3D).

SHH protein strongly expresses in DLBCL cells but not in less aggressive lymphomas, CLL and normal germinal center B cells ${ }^{14}$. We next studied the activation state of $\mathrm{SHH}$ signaling in vivo in a syngeneic B cell lymphoma model described in Fig. 1g. Twelve days of PN-1 treatment markedly reduced mRNA levels of GLI1 and SHH (Fig. 2gi). Angiogenesis is associated with aggressive subtypes of B cell lymphomas ${ }^{15}$. To determine the impact of $\mathrm{PN}-1$ on angiogenesis, we used CD31 staining in mice grafted with A20 lymphoma cells. Lymphomas grown with exogenous PN-1 recombination protein had lower vascular density (Figs. $2 \mathrm{~h}$ and S4). Residual blood vessels had larger diameters than controls. There was also less in situ protein levels of $S H H$-signaling molecules in tissues treated with PN-1 shown by immune blotting of whole tissue lysates and by immune-histochemistry (Fig. 2gii-h). Thus, PN-1 appears having an anti-angiogenic role in B cell lymphoma.

Interestingly, $M M P 9$ expression and $\mathrm{SHH}$ signaling are both known of regulating the growth and survival of 


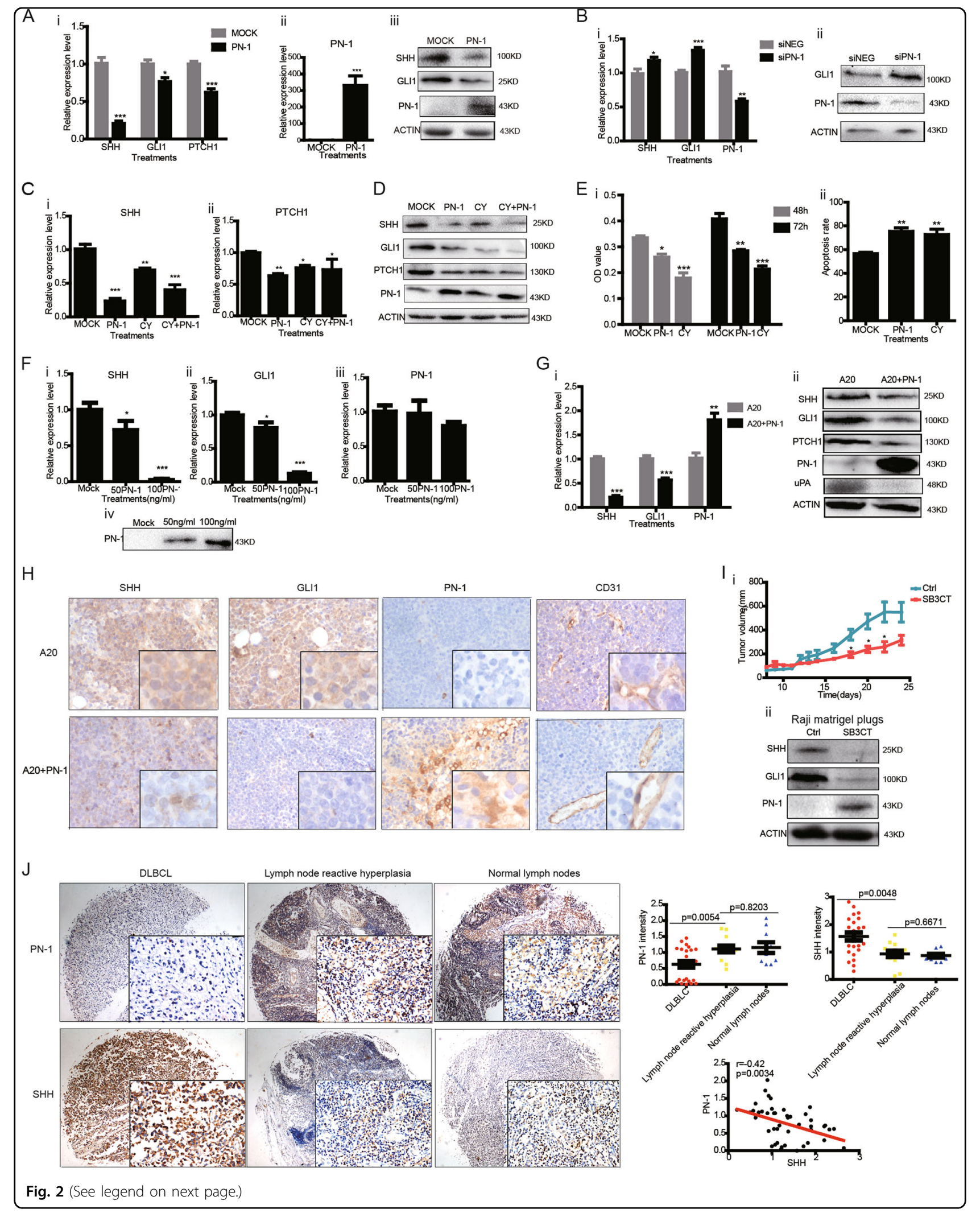


Fig. 2 PN-1 inhibits SHH signaling and angiogenesis of B cell lymphoma. a Real-time PCR (i-ii) and immunoblotting (iii) were applied to determine the expression of PN1, GLI1, PTCH1, and SMO in Raji cells transfected with a PN1 expression plasmid or empty vector $(2 \mu \mathrm{g})$ for $24 \mathrm{~h}(\mathrm{~N}=5$, ttest, ${ }^{*} P<0.05$; ${ }^{* *} P<0.001$ ). b Raji cells transfected with 40 pmol siRNA PN1 (siPN1) or control siRNA (siNEG) were measured for $P N 1$, SHH, or GL11 transcript levels using qRT-PCR (i) and protein levels by immune-blotting (ii) $\left(N=5 ; t\right.$ test, $\left.{ }^{*} P<0.05 ;{ }^{* *} P<0.01 ;{ }^{* * *} P<0.001\right)$. c Transcriptional levels of SHH (i), PTCH1 (ii) in Raji cells transfected with pcDNA3-PN1 plasmid $(2 \mu \mathrm{g})$ or treated with a SMO inhibitor cyclopamine $(20 \mu \mathrm{M})$ or both for $24 \mathrm{~h}(\mathrm{~N}=$ 6; one-way ANOVA; ${ }^{*} P<0.05$; ${ }^{* *} P<0.01$; ${ }^{* *} P<0.001$ ). $\mathbf{d}$ Immune-blotting of $\mathrm{SHH}$-signaling pathway molecules and PN-1 from Raji cells treated as indicated above. e Cell proliferation (i) was analyzed by CCK-8 proliferation assay after 48 and $72 \mathrm{~h}$ culture of cells treated with PN1 transfection $(2 \mu \mathrm{g})$ or cyclopamine $(20 \mu \mathrm{M})$; apoptosis (ii) was determined by flow cytometry via Annexin-PI staining at $24 \mathrm{~h}$ as indicated above ( $N=4$; one-way ANOVA ${ }^{*} P<0.05 ;{ }^{* *} P<0.01$; $\left.{ }^{* *} P<0.001\right)$. f A20 cells $\left(2 \times 10^{6}\right)$ were treated with mouse PN-1 recombinant protein at 50 or $100 \mathrm{ng} / \mathrm{ml}$ and mRNA transcripts SHH (i), GLI1 (ii), and PN1(iii) were measured by $q$-RT-PCR ( $N=3$; one-way ANOVA $*<<0.05$; $\left.{ }^{* *} P<0.001\right)$. Immune-blotting of PN-1 protein was performed using A20 cell conditioned medium (iv); g real-time PCR (i) for PN1, SHH, and GLI1 in A20 xenografts with or without PN-1 (10 $\mu M)(N=5 ; t$ test, ${ }^{* *} P<0.01$; $\left.{ }^{* *} P<0.001\right)$; A20 xenografts with or without PN-1 $(10 \mu \mathrm{M})$ were blotted via immune blotting (ii). $\mathbf{h}$ immune-histochemistry (brown) for SHH-signaling cascades and PN-1 in A20 xenograft tumor. DAB-stained for the angiogenesis marker CD31 (brown). Blue stain is hemotoxylin nuclear staining; i(i) Subcutaneous tumor volumes from NOD/SCID mice implanted with Raji cells $\left(5 \times 10^{6}\right)$ mixed with PBS or Matrigel with or without SB3CT $(7.5 \mu \mathrm{M})$ were measured and plotted $\left(N=3\right.$; $t$ test $\left.{ }^{*} P<0.05\right)$. (ii) Immune blots of PN-1, SHH, and GLI1 in Raji Nod/SCID xenografts with or without SB3CT $(7.5 \mu \mathrm{M})$. j DAB staining of $\mathrm{PN}-1$ and $\mathrm{SHH}$ in human lymphoid tissues. Measurement of staining intensity compared with lymph nodes from normal $(N=10)$, lymph node reactive hyperplasia $(N=13)$, or $\mathrm{DLBCL}$-infiltrated lymph nodes $(N=24)$. One-way ANOVA performed. Correlation between $\mathrm{PN}-1$ and $\mathrm{SHH}$ calculated using Spearman $r$ value

diverse NHL cells ${ }^{5,7,15}$. Our data suggest that PN1 might be a key player who connects these two important molecules. We subcutaneously injected Raji cells into NOD/SCID mice. Adding $7.5 \mu \mathrm{M}$ SB3CT to the Matrigel plug inhibited growth of the Raji cells compared with control Matrigel plugs (Fig. 2i-i). At $24 \mathrm{~d}$ the tumor volume of treated mice were $260 \mathrm{~mm}^{3}$ versus $550 \mathrm{~mm}^{3}$ in controls ( $\mathrm{p}<0.05$; Fig. $2 \mathrm{i}-\mathrm{i})$. SB3CT treatment resulted in increased levels of PN-1 and reduced levels of $\mathrm{SHH}$ and GLI (Fig. 2i-ii). These data suggest that PN-1 inhibition of SHH signaling is governed by MMP-9, presumably attributed to increased stability of PN-1 upon chemical inhibition of MMP-9 activity by SB3CT.

Finally, because of the heterogeneity of DLBCL, we determined the levels of SHH and PN-1 in normal lymph nodes, reactive lymph nodes and lymph nodes infiltrated with DLBCL cells. SHH protein localizes predominantly to the cytoplasm of the lymphoma cells but sparse in the microenvironment (Fig. 2j). PN-1 expression was evident in the stroma and vessels of normal and reactive lymph nodes but less so in lymph nodes infiltrated by DLBCL cells (Fig. 2j). There was an inverse correlation between PN-1 and SHH levels in tissues infiltrated by DLBCL cells $(r=-0.42 ; p=0.0034)$. These data suggest lymph nodes infiltrated by DLBCL cells had variable expression of both proteins, whereas normal lymph nodes were less variable. This finding is not surprising in view of the considerable heterogeneity of DLBCL. Gene chip analyses from 414 newly diagnosed cases of DLBCL revealed MMP9 and $P L A U$ in the stromal signature, suggesting genes encoding ECM proteases such as MMP9 might play critical roles on progression of B cell lymphoma ${ }^{5}$. However, considerable data suggest a striking heterogeneity in gene expression profiles and clinical outcomes of DLBCL, posing a challenge for clinical management. Consequently, molecular regulation of MMP-9, $\mathrm{PN}-1$, and $\mathrm{SHH}$ may be different across DLBCL subtypes. SHH is produced by dendritic cells in the germinal center (GC) protecting germinal center B cell from apoptosis ${ }^{15}$. DLBCL cells secrete and respond to endogenous $\mathrm{SHH}$ ligands, implicating an autocrine $\mathrm{SHH}$-signaling loop ${ }^{14}$. Our data from immune histo-chemical staining supports this concept.

Taken together, we present data supporting a new pathway by which $P N 1$ expression regulates lymphoma cell survival by inhibiting $\mathrm{SHH}$ signaling, an important survival signaling in the growth of B cell lymphomas. PN1 regulates $\mathrm{SHH}$ signaling by reducing $S H H$ transcripts and downstream effectors and by inhibiting angiogenesis. Such a regulation is also reflected by the inverse correlation between $\mathrm{PN}-1$ and $\mathrm{SHH}$ proteins demonstrated in human DLBCL tissue microarray samples. The crosstalk between MMP-9, PN-1, and SHH signaling supports an inhibitory role of $\mathrm{PN}-1$ in $\mathrm{B}$ cell lymphoma progression. MMP-9 and PN-1 may serve as potential molecular targets for combination therapies in DLBCL, in particular for those lymphomas with high expression of MMP9.

\section{Acknowledgements}

DX and YD are funded by Natural Science Foundation of China (81372326; 81273252). JFA and RPG acknowledge support from the National Institute of Health Research (NIHR) Biomedical Research Centre funding scheme.

\section{Author details}

'Department of Haematology, Tongji Hospital, Tongji Medical College, Huazhong University of Science and Technology, Hubei, China. ${ }^{2}$ Department of Endocrinology, Tongji Hospital, Tongji Medical College, Huazhong University of Science and Technology, Hubei, China. ${ }^{3}$ Gray Institute of Radiation Oncology and Biology, Medical Science Division, University of Oxford, Oxford, UK. ${ }^{4}$ Department of Haematology, Hammersmith Hospital, Imperial College Healthcare NHS Trust, Imperial College London, Du Cane road, London W12 $\mathrm{OHS}, \mathrm{UK}$

\section{Conflict of interest}

The authors declare that they have no conflict of interest. 


\section{Publisher's note}

Springer Nature remains neutral with regard to jurisdictional claims in published maps and institutional affiliations.

Supplementary Information accompanies this paper at https://doi.org/ 10.1038/s41408-018-0063-x.

Received: 16 October 2017 Revised: 07 January 2018 Accepted: 15 January 2018

Published online: 26 February 2018

\section{References}

1. International Non-Hodgkin's Lymphoma Prognostic Factors Project. A predictive model for aggressive non-Hodgkin's lymphoma. N. Engl. J. Med 329, 987-994 (1993)

2. Coiffier, B. et al. CHOP chemotherapy plus rituximab compared with $\mathrm{CHOP}$ alone in elderly patients with diffuse large-B-cell lymphoma. N. Engl. J. Med. 346, 235-242 (2002)

3. Younes, A. Beyond chemotherapy: new agents for targeted treatment of lymphoma. Nat. Rev. Clin. Oncol. 8, 85-96 (2011).

4. Alizadeh, A. A. et al. Distinct types of diffuse large B-cell lymphoma identified by gene expression profiling. Nature 403, 503-511 (2000).
5. Lenz, G. et al. Stromal gene signatures in large-B-cell lymphomas. N. Engl. J. Med. 359, 2313-2323 (2008)

6. Joyce, J. A. \& Pollard, J. W. Microenvironmental regulation of metastasis. Nat. Rev. Cancer 9, 239-252 (2009).

7. Sakata, K. et al. Expression of matrix metalloproteinase 9 is a prognostic factor in patients with non-Hodgkin lymphoma. Cancer 100, 356-365 (2004).

8. Kruger, A. et al. Antimetastatic activity of a novel mechanism-based gelatinase inhibitor. Cancer Res. 65, 3523-3526 (2005).

9. Xu, D. et al. Matrix metalloproteinase-9 regulates tumor cell invasion through cleavage of protease nexin-1. Cancer Res. 70, 6988-6998 (2010).

10. McKee, C. M., Xu, D. \& Muschel, R. J. Protease nexin 1: a novel regulator of prostate cancer cell growth and neo-angiogenesis. Oncotarget 4, 1-2 (2013).

11. McKee, C. M et al. Protease nexin 1 inhibits hedgehog signalling in prostate adenocarcinoma. J. Clin. Invest. 122, 4025-4036 (2012).

12. Xu, D. et al. Novel MMP-9 substrates in cancer cells revealed by a label-free quantitative proteomics approach. Mol. Cell. Proteomics 7, 2215-2228 (2008).

13. McKee, C. M. et al. Protease nexin 1 induces apoptosis of prostate tumor cells through inhibition of X-chromosome-linked inhibitor of apoptosis protein. Oncotarget 6, 3784-3796 (2015).

14. Singh, R. R. et al. Hedgehog signalling pathway is activated in diffuse large Bcell lymphoma and contributes to tumor cell survival and proliferation. Leukemia 24, 1025-1036 (2010).

15. Dierks, C. et al. Essential role of stromally induced hedgehog signalling in B-cell malignancies. Nat. Med. 13, 944-951 (2007). 